\title{
Self-management for adults with epilepsy: Aggregate Managing Epilepsy Well Network findings on depressive symptoms
}

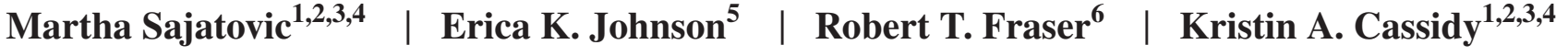 \\ Hongyan Liu ${ }^{2,3,4}$ | Dilip K. Pandey ${ }^{7}$ | Rakale C. Quarells ${ }^{8}$ | Peter Scal ${ }^{9}$ | \\ Samantha Schmidt $^{10}$ | Ross Shegogi ${ }^{11}$ | Tanya M. Spruill ${ }^{12}$ | Mary R. Janevic ${ }^{13}$ | \\ Curtis Tatsuoka $^{2,3,4}$ | Barbara C. Jobst ${ }^{10}$ \\ ${ }^{1}$ Department of Psychiatry, University Hospitals Cleveland Medical Center, Cleveland, Ohio \\ ${ }^{2}$ Department of Neurology, University Hospitals Cleveland Medical Center, Cleveland, Ohio \\ ${ }^{3}$ Case Western Reserve University School of Medicine, Cleveland, Ohio \\ ${ }^{4}$ Neurological and Behavioral Outcomes Center, University Hospitals Cleveland Medical Center, Cleveland, Ohio \\ ${ }^{5}$ Health Promotion Research Center, University of Washington, Seattle, Washington \\ ${ }^{6}$ Department of Rehabilitation Medicine, University of Washington, Seattle, Washington \\ ${ }^{7}$ Department of Neurology and Rehabilitation, University of Illinois College of Medicine at Chicago, Chicago, Illinois \\ ${ }^{8}$ Department of Community Health and Preventive Medicine, Morehouse School of Medicine, Atlanta, Georgia \\ ${ }^{9}$ Department of Pediatrics and Adolescent Health, University of Minnesota, Minneapolis, Minnesota \\ ${ }^{10}$ Department of Neurology, Geisel School of Medicine at Dartmouth, Dartmouth College, Lebanon, New Hampshire \\ ${ }^{11}$ University of Texas Health Science Center at Houston School of Public Health, Houston, Texas \\ ${ }^{12}$ Department of Population Health, New York University School of Medicine, New York, New York \\ ${ }^{13}$ Center for Managing Chronic Disease, University of Michigan, Ann Arbor, Michigan
}

\section{Correspondence}

Martha Sajatovic, Department of Psychiatry, W. O. Walker Bldg, 7th Floor, 10524 Euclid Avenue, Cleveland, OH 44106, USA.

Email: martha.sajatovic@uhhospitals.org

\begin{abstract}
Objective: To assess depressive symptom outcomes in a pooled sample of epilepsy self-management randomized controlled trials (RCTs) from the Managing Epilepsy Well (MEW) Network integrated research database (MEW DB).

Methods: Five prospective RCTs involving 453 adults with epilepsy compared self-management intervention $(\mathrm{n}=232$ ) versus treatment as usual or wait-list control outcomes $(n=221)$. Depression was assessed with the nine-item Patient Health Questionnaire. Other variables included age, gender, race, ethnicity, education, income, marital status, seizure frequency, and quality of life. Follow-up assessments were collapsed into a visit 2 and a visit 3; these were conducted postbaseline.

Results: Mean age was 43.5 years $(\mathrm{SD}=12.6)$, nearly two-thirds were women, and nearly one-third were African American. Baseline sample characteristics were mostly similar in the self-management intervention group versus controls. At followup, the self-management group had a significantly greater reduction in depression
\end{abstract}

The aggregate data were derived from individual MEW Network research studies with approval by the institutional review board of University Hospitals Cleveland Medical Center (principal investigator: Martha Sajatovic). This publication is a product of a Health Promotion and Disease Research Center supported by Cooperative Agreement Number 5U48DP005030 from the Centers for Disease Control and Prevention. 
compared to controls at visit $2(P<.0001)$ and visit $3(P=.0002)$. Quality of life also significantly improved in the self-management group at visit $2(P=.001)$ and visit $3(P=.005)$.

Significance: Aggregate MEW DB analysis of five RCTs found depressive symptom severity and quality of life significantly improved in individuals randomized to selfmanagement intervention versus controls. Evidence-based epilepsy self-management programs should be made more broadly available in neurology practices.

\section{K E Y W O R D S}

depression, epilepsy, quality of life, seizures, self-management

\section{$1 \mid$ INTRODUCTION}

Epilepsy self-management is an approach that helps people with epilepsy learn skills to help them better manage their epilepsy and its effect on daily life. Three broad areas targeted are treatment management, seizure management, and lifestyle management. ${ }^{1-8}$ In 2007, the US Centers for Disease Control and Prevention (CDC) established the Prevention Research Centers' Managing Epilepsy Well (MEW) Network to develop, test, and disseminate epilepsy self-management interventions. ${ }^{3,9}$

The MEW Network has developed an integrated database (MEW DB) that pools data from epilepsy self-management studies to conduct aggregate and secondary analysis. ${ }^{10,11} \mathrm{~A}$ recent analysis from the MEW DB that examined correlates of depressive symptoms assessed with the nine-item Patient Health Questionnaire (PHQ-9) found more severe depression in those with poorly controlled seizures. ${ }^{12}$ Other studies demonstrate that depression in epilepsy is common and associated with powerful negative effects including worse seizure control, poor quality of life, and premature mortality due to suicide. $^{13-15}$

A recent literature review of psychological treatments in people with epilepsy found that one-third of patients receiving cognitive behavioral therapy (CBT) interventions, compared to $10 \%$ of controls, could be considered "reliably improved." 16 However, given the great number of undertreated people with epilepsy who also have depression, the development of additional psychological approaches, including alternatives to CBT, is warranted. There is considerable conceptual overlap among psychological approaches intended to help improve health outcomes among people with epilepsy. For example, both CBT and epilepsy self-management interventions include a focus on improving emotional regulation and the development of personal coping strategies that address solving current problems. ${ }^{16,17}$ Although there is still a relative paucity of randomized controlled trials (RCTs) focused on epilepsy self-management interventions, ${ }^{18}$ the

\section{Key Points}

- This report analyzed pooled data from five selfmanagement randomized controlled trials from the Managing Epilepsy Well Network

- The sample of 453 adults had a mean age of 43.5 years, nearly two-thirds were women, and nearly one-third were African American

- At follow-up, self-management intervention patients experienced significantly reduced depressive symptoms compared to controls

- Evidence-based epilepsy self-management programs should be made more broadly available in neurology practices

CDC-supported MEW Network website describes evidence-based epilepsy self-management intervention such as the HOBSCOTCH (Home-Based Self-Management and Cognitive Training Changes Lives) approach intended to improve cognitive problems in adults with epilepsy and the TIME (Targeted Self-Management for Epilepsy and Mental Illness) approach targeting adults with epilepsy and comorbid mental health conditions, as well as emerging research for self-management interventions that are still in development. ${ }^{8}$ Given the MEW Network's focus on depression comorbidity, a number of sites conducting RCTs in epilepsy self-management interventions have the PHQ-9 as a primary or secondary outcome. All MEW Network RCTs have used a treatment as usual or wait-list comparison control, providing relative homogeneity in study design.

Given the critical need for managing depressive symptoms in epilepsy and the limited benefit with standardized psychological approaches such as $\mathrm{CBT},{ }^{17}$ this aggregate analysis was conducted to address the broad question of whether a curriculum-driven epilepsy self-management intervention can improve depression outcomes in people with epilepsy. 
This is a first-ever evaluation of the MEW DB longitudinal data to examine depressive symptom severity outcomes in a pooled sample of epilepsy self-management intervention RCTs. We hypothesized that individuals randomized to a self-management intervention would have greater reduction in depressive symptom severity over time compared to controls.

\section{MATERIALS AND METHODS}

\section{$2.1 \mid$ Overview}

This analysis of longitudinal data included 453 adults with epilepsy enrolled in five MEW Network prospective randomized controlled epilepsy self-management intervention trials. Details describing the MEW Network, including data harmonization, have been described elsewhere. ${ }^{9}$ The aggregate data were derived from individual MEW Network research studies with approval by the institutional review board of University Hospitals Cleveland Medical Center. Only RCTs that included use of the PHQ-9 administered in a longitudinal manner were included in this analysis. The deidentified data used for the analysis were obtained from the HOBSCOTCH trial at Dartmouth-Hitchcock Medical Center, ${ }^{5}$ the PACES (Program of Active Consumer Engagement in SelfManagement) trial from the University of Washington, the FOCUS (Figure Out the Problem, Observe Your Routine, Connect Your Observations and Choose a Change Goal, Undertake a Change Strategy, and Study the Results) trial from the University of Michigan, and the TIME and SMART (Self-Management for People With Epilepsy and a History of Negative Health Events) trials from Case Western Reserve University. All RCT participants were adults at least 18 years of age or older. All studies had a self-reported diagnosis of epilepsy except where noted in the specific study descriptions below. None of the studies required a depression diagnosis or a specific depression severity threshold for study inclusion. All study participants expressed informed consent for participation in the respective studies.

\section{2 | Description of each study}

\subsection{1 | HOBSCOTCH}

Study design: Prospective RCT.

Inclusion criteria: Adult patients with a diagnosis of epilepsy (confirmed by clinical evidence or ancillary studies). Epilepsy was controlled or uncontrolled but without severe intellectual disability. Participants had subjective memory complaints defined as scores of $\leq 7$ on a subset of cognition questions of the Quality of Life in Epilepsy
(QOLIE-31). (Subset questions were normalized to a score between 0 and 10.)

Sample: Sixty-six adults, age $=18-65$ years.

Description of the intervention: Self-management intervention delivered one-on-one, primarily by telephone. Combines problem-solving therapy with memory strategies. ${ }^{19}$

Intervention comparator: Twenty-four-week wait-list control.

Key outcomes: Quality of life and objective memory. Depressive symptom severity using the PHQ-9 was assessed as a covariate. In the original study primary outcome report, depression scores among the treatment cohorts showed improvement but did not reach statistical significance. ${ }^{5}$

Total study duration, timing of research assessments: Twenty-four-week study, assessments at baseline, 8-10 weeks (visit 2), and 24 weeks (visit 3).

\subsection{2 | PACES $^{6}$}

Study design: Prospective RCT.

Inclusion criteria: Epilepsy without substantive cognitive impairment. Inclusion criteria also included having active (seizure within the past 6 months) and chronic (at least 6 months since diagnosis) epilepsy.

Sample: Two hundred eighty-three adults, age $\geq 18$ years.

Description of the intervention: In-person, 8-week medical and psychosocial self-management group intervention that is focused on improving medical and psychosocial management, problem-solving, and behavioral activation.

Intervention comparator: Treatment as usual.

Key outcomes: The primary study outcomes were epilepsy self-efficacy, epilepsy self-management, and goal attainment. Depression (PHQ-9) was assessed as a secondary outcome. In the original study primary outcome report, depressive symptoms were significantly improved at program completion. ${ }^{6}$

Total study duration, timing of research assessments: Twenty-four-week study. Research assessments were conducted at baseline (start of program), immediately postintervention ( 8 weeks/visit 2 ), and at 24 weeks postprogram (visit $3)$.

\subsection{3 | FOCUS}

Study design: Prospective RCT. Study outcomes have not been published.

Inclusion criteria: Adults with epilepsy for at least 1 year, taking antiepileptic medication daily and being able to identify a support person willing to participate.

Sample: One hundred thirty adults, age $\geq 21$ years.

Description of the intervention: Eight-week hybrid inperson workshop and telephone coaching program that developed self-regulation skills in both adults with epilepsy and a key friend or family member who provides support. 
Intervention comparator: Control group members received written patient education materials on topics known to impact quality of life for people with epilepsy (eg, sleep and stress) and information on regional and national epilepsy resources.

Key outcomes: The primary study outcome was the 31item QOLIE-31. Depression, using the PHQ-9, was assessed as a secondary outcome. In the original study analysis, no significant differences between the intervention and control groups were found in pre-post changes in depression.

Total study duration, timing of research assessments: Forty-week study. Active intervention period was 10 weeks. Research assessments (telephone surveys) were conducted at baseline (prior to start of program) and approximately 40 weeks from baseline (visit 2).

\subsection{4 | SMART $^{20}$}

Study design: Prospective 24-week RCT. The RCT was followed by a 12-month extension follow-up phase.

Inclusion criteria: Epilepsy diagnosis and occurrence of a negative health event (NHE) defined as at least one seizure, emergency room visit, hospitalization, or self-harm attempt within the past 6 months.

Sample: One hundred twenty adults, age $\geq 18$ years.

Description of the intervention: Remotely delivered (Web or telephone) nurse + peer educator group-format self-management intervention focused on managing seizures, stress, and life-style to optimize health functioning.

Intervention comparator: Twenty-four-week wait-list control.

Key outcomes: The primary study outcome was change in NHE counts from baseline to follow-up assessments at week 12 and week 24. Depression using the PHQ-9 was assessed as a secondary outcome. In the original study primary outcome report, depression scores were significantly improved in SMART versus controls. ${ }^{20}$

Total study duration, timing of research assessments: Only the 24-week RCT data were used for this analysis. Research assessments were done at baseline, 10 weeks (visit 2), and 24 weeks (visit 3).

\subsection{5 | TIME $^{7}$}

Study design: Prospective RCT.

Inclusion criteria: Epilepsy and the presence of comorbid serious mental illness defined as schizophrenia, bipolar disorder, or major depression. Depressive symptoms were not required for inclusion.

Sample size, mean age: Forty-four adults, age $\geq 18$ years.

Description of the intervention: In-person, nurse and peer educator led group-format intervention to improve both mood and epilepsy outcomes.
Intervention comparator: Treatment as usual.

Key outcomes: The primary study outcome was depressive symptom severity assessed with the Montgomery-Asberg Depression Rating Scale. ${ }^{21}$ Depressive symptoms using the PHQ-9 were assessed as a secondary outcome. In the original study primary outcome report, depression scores were significantly improved in TIME versus controls. ${ }^{7}$

Total study duration, timing of research assessments: Sixteen-week study. Research assessments were conducted at baseline, 12 weeks (visit 2), and 16 weeks (visit 3) follow-up.

\section{3 | Measures}

Variables assessed in the studies included age, gender, race, ethnicity, educational level, income, marital/relationship status, seizure frequency, and two standardized measures that evaluated depressive symptom severity and epilepsy-related quality of life.

\section{4 | Depressive symptom severity}

Depressive symptom severity was assessed using the PHQ-9, a widely used and validated self-rated depression scale. ${ }^{22}$ The PHQ-9 incorporates Diagnostic and Statistical Manual of Mental Disorders diagnostic criteria, with scores ranging from 0 to 27. Items are scored on a 0-3 continuum, with higher scores indicating worse depressive symptom severity. Based on total PHQ-9 scores, there are several well-documented groups of depressive severity: $1-4=$ minimal depression, 5-9 = mild depression, 10-14 = moderate depression, 15-19 = moderately severe depression, and 20-27 = severe depression.

\section{5 | Quality of life}

Quality of life was assessed with an adapted version of the 10-item QOLIE (QOLIE-10) instrument, a self-administered questionnaire developed from the original QOLIE- $89 .{ }^{23}$ The QOLIE-10 has good test-retest reliability and correlates well with longer versions of this instrument. ${ }^{24} \mathrm{~A} 31$-item version of the QOLIE (QOLIE-31) and a patient-weighted version of the QOLIE (QOLIE-P) include the same 10 questions but have slightly different scoring ranges (1-6, 1-4) on three items. Given the slightly different versions of the questions across studies in the MEW DB, scores were calibrated to yield a total possible score range of 1-5, with lower scores indicating better quality of life and fewer problems related to epilepsy.

\subsection{Data cleaning and harmonization}

All MEW-DB data are linked following a study protocol and a data dictionary with labels for each variable. Study datasets were 
first evaluated to confirm that data dictionary variables clearly delineated dataset content and assessment timing. As has been described elsewhere, data mapping was done to allow integration between study-specific variables and the MEW common terminology system, and involved reconciling differences in both data values and interval values used to categorize the data elements. ${ }^{10}$ For seizure frequency, we "prorated" counts based upon the time interval being assessed to derive a past 30-day seizure frequency. For example, if the original study's seizure count data were derived from a 90-day period, we would divide the count by 3 to calculate a 30-day seizure frequency. For the longitudinal component of the analysis, given that our intent was to evaluate the trajectories of depressive symptom severity over time, we collapsed data collection follow-up time-points into two groups of follow-up assessments. Visit 2 was the first assessment conducted after completion of the self-management intervention, and visit 3 was the second assessment done after the self-management intervention. Thus, visit 2 was conducted
TA B L E 1 Baseline demographic and clinical variables among people with epilepsy randomized to self-management intervention compared to controls

\begin{tabular}{|c|c|c|c|c|}
\hline Variable & $\begin{array}{l}\text { Total } \\
\text { sample, } \\
\mathrm{N}=453\end{array}$ & $\begin{array}{l}\text { Intervention } \\
\text { sample, } \mathbf{n}=\mathbf{2 3 2}\end{array}$ & $\begin{array}{l}\text { Control } \\
\text { sample, } \\
\text { n= 221 }\end{array}$ & $P$ \\
\hline Age, mean (SD) & $43.5(12.6)$ & $44.5(12.5)$ & $42.4(12.6)$ & $.0662^{\mathrm{a}}$ \\
\hline Female, n (\%) & $288(63.7 \%)$ & $147(63.6 \%)$ & $141(63.8 \%)$ & $.5893^{\mathrm{b}}$ \\
\hline \multicolumn{5}{|l|}{ Race, n (\%) } \\
\hline White & $232(59.9 \%)$ & $110(58.5 \%)$ & $122(61.3 \%)$ & \multirow[t]{3}{*}{$.6434^{\mathrm{b}}$} \\
\hline Black/African American & $123(31.8 \%)$ & $60(31.9 \%)$ & $63(31.7 \%)$ & \\
\hline Other & $32(8.3 \%)$ & $18(9.6 \%)$ & $14(7 \%)$ & \\
\hline \multicolumn{5}{|l|}{ Ethnicity, n (\%) } \\
\hline Not Hispanic & $356(92.7 \%)$ & $170(91.9 \%)$ & $186(93.5 \%)$ & \multirow[t]{2}{*}{$.5530^{\mathrm{b}}$} \\
\hline Hispanic & $28(7.3 \%)$ & $15(8.1 \%)$ & $13(6.5 \%)$ & \\
\hline \multicolumn{5}{|l|}{ Education, n (\%) } \\
\hline High school or less & $141(31.4 \%)$ & $73(31.9 \%)$ & $68(30.9 \%)$ & \multirow[t]{2}{*}{$.8250^{\mathrm{b}}$} \\
\hline At least some college & $308(68.6 \%)$ & $156(68.1 \%)$ & $152(69.1 \%)$ & \\
\hline \multicolumn{5}{|l|}{ Income, n (\%) } \\
\hline$<\$ 25 \mathrm{~K}$ & $227(69.6 \%)$ & $114(70.8 \%)$ & $113(68.5 \%)$ & \multirow[t]{3}{*}{$.8273^{\mathrm{b}}$} \\
\hline$\$ 25-50 \mathrm{~K}$ & $40(12.3 \%)$ & $20(12.4 \%)$ & $20(12.1 \%)$ & \\
\hline$>\$ 50 \mathrm{~K}$ & $59(18.1 \%)$ & $27(16.8 \%)$ & $32(19.4 \%)$ & \\
\hline \multicolumn{5}{|l|}{ Marital status, n (\%) } \\
\hline Married or partnered & $73(29.7 \%)$ & $46(38.0 \%)$ & $27(21.6 \%)$ & $.0048^{\mathrm{b}}$ \\
\hline Other & $173(70.3 \%)$ & $75(62.0 \%)$ & $98(78.4 \%)$ & \\
\hline $\begin{array}{l}\text { 30-day seizure frequency, } \\
\text { mean (SD) }\end{array}$ & $4.5(21.0)$ & $4.4(24.3)$ & $4.5(16.8)$ & $.4576^{\mathrm{c}}$ \\
\hline QOLIE-10, mean (SD) & $2.9(0.9)$ & $2.9(0.9)$ & $2.8(0.8)$ & $.6255^{\mathrm{a}}$ \\
\hline PHQ-9, mean (SD) & $9.2(6.5)$ & $8.9(6)$ & $9.4(6.9)$ & $.4635^{\mathrm{b}}$ \\
\hline Minimal depression, $\mathrm{n}(\%)$ & $176(20.1 \%)$ & $93(20.0 \%)$ & $83(20.2 \%)$ & \\
\hline Mild depression, n (\%) & $288(32.8 \%)$ & $172(37.0 \%)$ & $116(28.2 \%)$ & \\
\hline Moderate depression, n (\%) & $195(22.2 \%)$ & $105(22.6 \%)$ & $90(21.8 \%)$ & \\
\hline $\begin{array}{l}\text { Moderately severe depres- } \\
\text { sion, } \mathrm{n}(\%)\end{array}$ & $134(15.3 \%)$ & $70(15.1 \%)$ & $62(15.5 \%)$ & \\
\hline Severe depression, $\mathrm{n}(\%)$ & $84(9.6 \%)$ & $25(5.4 \%)$ & $59(14.3 \%)$ & \\
\hline
\end{tabular}

Note: Minimal depression: PHQ-9 total score $=1-4$; mild depression: PHQ-9 total score $=5-9$; moderate depression: PHQ-9 total score $=10-14$; moderately severe depression: PHQ-9 total score $=15-19$; severe depression: PHQ-9 total score $=20-27$.

Abbreviations: PHQ-9, nine-item Patient Health Questionnaire; QOLIE-10, 10-item Quality of Life in Epilepsy.

${ }^{\mathrm{a}} t$ test.

${ }^{\mathrm{b}}$ Chi-square test.

${ }^{\mathrm{c}}$ Mann-Whitney $U$ test. 
8-12 weeks after baseline (except for FOCUS, which had a single follow-up visit at 36-40 weeks), whereas visit 3 was conducted 16-36 weeks after baseline. Because the FOCUS study had only one follow-up assessment, the follow-up visit was considered the visit 2 data collection point.

\subsection{Data analysis}

Statistical analysis was performed using SAS 9.4 (SAS Institute). Descriptive analyses characterized the baseline sample and examined change over time in PHQ-9. Longitudinal mixed models from baseline to visit 2 and visit 3 were conducted. A type I error rate of 0.05 was used. To validate the pooled longitudinal PHQ-9 total findings and confirm that they were not contingent upon a single study, a series of $t$ tests for the change from baseline to visit 2 and change from baseline to visit 3 were conducted by leaving out each one of the studies, one at a time. To examine the relationship between variables at baseline and change in PHQ-9 over time, mixed model analyses were conducted in the combined group of intervention + controls. Given the known relationship between quality of life and depressive symptom severity, the association between a 1-point change in QOLIE-10 and PHQ-9 was also quantified.

\section{3 | RESULTS}

\section{1 | Baseline sample}

Table 1 shows characteristics of all individuals with epilepsy in the pooled sample $(\mathrm{N}=453)$, as well as by treatment status: self-management intervention group $(\mathrm{n}=232)$ versus controls $(\mathrm{n}=221)$. Mean age of the combined sample was 43.5 years (SD = 12.6), with nearly two-thirds being women and nearly one-third being African American (Table 1). Although the majority of the combined sample had some education beyond high school $(68.6 \%, \mathrm{n}=308)$, the majority were living in restricted financial circumstances, with $69.6 \%(n=227)$ having an annual income below US \$25 000. Most demographic and clinical characteristics were similar between the intervention and control groups. Only marital status was statistically different between the intervention and control groups, with slightly more individuals in the intervention group $(38 \%, \mathrm{n}=46)$ versus controls $(21.6 \%, \mathrm{n}=27)$ being married or partnered.

\subsection{Change in depressive symptom severity over time}

Tables 2 and 3 show the change over time in total unadjusted mean PHQ-9 scores in the self-management intervention versus control groups and in each of the five RCTs separately. As seen in Figure 1, in the pooled sample over the three assessment time-points, individuals randomized to self-management intervention had a significantly greater reduction in total depressive symptom severity compared to controls at visit $2(P<.0001)$ and at visit $3(P=.0002)$. As noted in Table 4 , the validation exercise in which each study was omitted one at a time and the remainder evaluated for change over time showed largely similar findings to the pooled analysis with respect to change in PHQ-9 totals between baseline and visits 2 and 3. In addition to examination of PHQ-9 as a continuous variable, we also examined the clinically relevant change in the sample proportion in the self-management intervention group versus the control group who improved from having a depressive symptom severity score of moderately depressed (PHQ-9 score $\geq 10$ ). There was no statistically significant difference in the percentage of the intervention group (41.3\%) versus control group (44.2\%) that had a PHQ-9 score of $\geq 10$ at baseline $(P=.537)$. However, at visit 3 , the percentages were significantly different $(P<.001)$, with only $24.8 \%$ of the intervention group having a PHQ-9 score $\geq 10$ versus $47.1 \%$ of controls.

\subsection{Association between demographic/ clinical variables and change in depressive symptom severity}

As noted in Table 5, quality of life was also significantly improved in self-management intervention versus controls at visit $2(P=.001)$ and visit $3(P=.005)$. Mixed-model analyses are shown in Table 6. For the combined sample, treatment assignment, visit time-point, educational level, and quality of life at baseline were all significantly associated with change in PHQ-9. Individuals randomized to the intervention had reduced depressive symptom levels over

\begin{tabular}{|c|c|c|c|c|}
\hline \multirow[b]{3}{*}{ Visit } & \multirow[b]{3}{*}{ Mean (SD) } & \multicolumn{2}{|c|}{ Treatment assignment } & \multirow[b]{3}{*}{$P$} \\
\hline & & Intervention & Control & \\
\hline & & Mean (SD) & Mean (SD) & \\
\hline Baseline, $\mathrm{N}=453$ & $9.2(6.5)$ & $8.9(6)$ & $9.4(6.9)$ & .4635 \\
\hline Visit $2, \mathrm{n}=398$ & $8.5(6.1)$ & $7.2(5.4)$ & $9.7(6.5)$ & $<.0001$ \\
\hline Visit $3, \mathrm{n}=255$ & $8.3(6.5)$ & $6.9(6.1)$ & $9.9(6.6)$ & .0002 \\
\hline
\end{tabular}

Abbreviation: PHQ-9, nine-item Patient Health Questionnaire.
T A B LE 2 Change in PHQ-9 total score over time in people with epilepsy randomized to self-management versus controls 
TABLE 3 Change in PHQ-9 total scores over time in five epilepsy selfmanagement randomized controlled trials

\begin{tabular}{|c|c|c|c|c|c|}
\hline \multirow[b]{3}{*}{ Study } & \multirow[b]{3}{*}{ Visit (week) ${ }^{b}$} & \multirow[b]{3}{*}{ Mean (SD) } & \multicolumn{2}{|c|}{ Treatment assignment } & \multirow[b]{3}{*}{$P^{\mathrm{a}}$} \\
\hline & & & Intervention & Control & \\
\hline & & & Mean (SD) & Mean (SD) & \\
\hline \multirow[t]{2}{*}{ FOCUS-RCT } & Baseline & $7.6(6.1)$ & $7.8(5.9)$ & $7.5(6.4)$ & \\
\hline & Visit $2(36-40)$ & $7.0(5.4)$ & $6.9(5.7)$ & $7.2(5.1)$ & .4051 \\
\hline \multirow[t]{3}{*}{ HOBSCOTCH } & Baseline & $9.4(5.9)$ & $9.6(5.8)$ & $9.2(6.3)$ & \\
\hline & Visit 2 (8-10) & $8.8(5.2)$ & $7.8(4.4)$ & $10.5(5.9)$ & .3395 \\
\hline & Visit 3 (24) & $8.1(5.9)$ & $7.1(5.4)$ & $9.6(6.4)$ & .1586 \\
\hline \multirow[t]{3}{*}{ PACES } & Baseline & $8.4(6.1)$ & $8.2(5.8)$ & $8.5(6.3)$ & \\
\hline & Visit 2 (12) & $7.2(5.4)$ & $5.2(4)$ & $8.8(5.9)$ & .0125 \\
\hline & Visit 3 (36) & $7(6.2)$ & $6.3(6.8)$ & $7.5(5.5)$ & .8277 \\
\hline \multirow[t]{3}{*}{ SMART } & Baseline & $10.7(7.2)$ & $10(6.6)$ & $11.5(7.8)$ & \\
\hline & Visit 2 (12) & $10(7.1)$ & $7.8(6.2)$ & $11.9(7.4)$ & .0050 \\
\hline & Visit 3 (24) & $9.1(7)$ & $7.3(6.6)$ & $10.8(7)$ & .1080 \\
\hline \multirow[t]{3}{*}{ TIME } & Baseline & $10.7(5.5)$ & $9.5(5.3)$ & $11.9(5.6)$ & \\
\hline & Visit 2 (12) & $11.2(5.6)$ & $9.2(5)$ & $13.2(5.7)$ & .1559 \\
\hline & Visit 3 (16) & $9.5(6.4)$ & $6.4(4.4)$ & $12.9(6.6)$ & .0131 \\
\hline
\end{tabular}

Abbreviations: PHQ-9, nine-item Patient Health Questionnaire; RCT, randomized controlled trial. ${ }^{\mathrm{a}}$ Change from baseline between intervention group versus control.

${ }^{b}$ Visits 2 and 3 were follow-up visits conducted after the completion of the epilepsy self-management program was completed. Visit 2 was conducted 8-12 weeks after baseline for all except FOCUS (which had a single follow-up visit conducted at 36-40 weeks). For all studies except FOCUS, which only had one follow-up timepoint, visit 3 was conducted 16-36 weeks after baseline.
The mean and standard error of PHQ-9

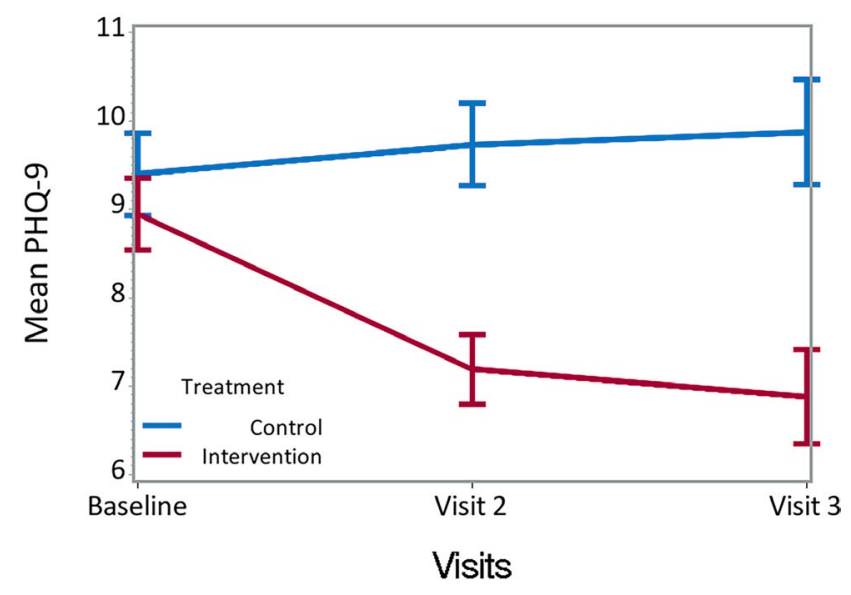

F I G U R E 1 Total mean score on nine-item Patient Health Questionnaire (PHQ-9) over time in self-management randomized participants versus controls. $P$ value at visit $2<.0001, P$ value at visit $3=.0002$

time as evidenced by the significant treatment-time interaction. Individuals with higher educational levels (at least some college) had lower endpoint PHQ-9 scores than those with lower education, with an average difference of 1.87 $(\mathrm{SD}=0.70)$. In examining only the sample of individuals randomized to control, lower education was associated with less improvement in depressive symptoms $(P=.033)$, whereas educational level was not significantly associated with change in depressive symptoms in the intervention group (data not shown). The relationship between quality of life and depression was significant, and a 4.9 increase in PHQ-9 (worse depression) corresponded to a one-point increase in QOLIE-10 (worse quality of life).

\section{DISCUSSION}

These analyses, taking advantage of a novel aggregate dataset from a CDC-sponsored research collaborative, ${ }^{8}$ investigated the relationship between participation in an epilepsy self-management intervention and depressive symptom severity outcomes over time. Analyses from five RCTs that all used a prospective design comparing self-management intervention versus treatment as usual or waitlist control suggests that depressive symptom severity is significantly reduced in people with epilepsy who participate in a self-management intervention program. Given the known association of depression with poor outcomes among people with epilepsy, ${ }^{1-8}$ the findings have important clinical implications along several dimensions.

A key clinical implication is the potential utility of selfmanagement intervention to advance care for depressed 


\begin{tabular}{|c|c|c|c|c|c|}
\hline \multirow[b]{3}{*}{ Dataset } & \multirow{3}{*}{$\begin{array}{l}\text { Difference } \\
\text { variable }\end{array}$} & \multirow[b]{3}{*}{ Mean (SD) } & \multicolumn{2}{|c|}{ Treatment assignment } & \multirow[b]{3}{*}{$P^{\mathrm{a}}$} \\
\hline & & & Intervention & Control & \\
\hline & & & Mean (SD) & Mean (SD) & \\
\hline \multirow[t]{2}{*}{$\begin{array}{l}\text { All datasets } \\
\text { included }\end{array}$} & $\begin{array}{l}\text { Diff base- } \\
\text { line vs V2 }\end{array}$ & $-0.6(5)$ & $-1.5(5)$ & $0.3(4.9)$ & .0003 \\
\hline & $\begin{array}{l}\text { Diff base- } \\
\text { line vs V3 }\end{array}$ & $-1.2(5.8)$ & $-2.1(5.5)$ & $-0.2(6)$ & .0120 \\
\hline \multirow[t]{2}{*}{$\begin{array}{l}\text { HOBSCOTCH } \\
\text { omitted }\end{array}$} & $\begin{array}{l}\text { Diff base- } \\
\text { line vs V2 }\end{array}$ & $-0.7(5)$ & $-1.7(5.1)$ & $0.3(4.8)$ & .0004 \\
\hline & $\begin{array}{l}\text { Diff base- } \\
\text { line vs V3 }\end{array}$ & $-1.2(5.9)$ & $-2.2(5.7)$ & $-0.4(6)$ & .0305 \\
\hline \multirow[t]{2}{*}{ FOCUS omitted } & $\begin{array}{l}\text { Diff base- } \\
\text { line vs V2 }\end{array}$ & $-0.5(5)$ & $-1.7(4.8)$ & $0.6(4.9)$ & .0001 \\
\hline & $\begin{array}{l}\text { Diff base- } \\
\text { line vs V3 }\end{array}$ & $-1.2(5.8)$ & $-2.1(5.5)$ & $-0.2(6)$ & .0120 \\
\hline \multirow[t]{2}{*}{ TIME omitted } & $\begin{array}{l}\text { Diff base- } \\
\text { line vs V2 }\end{array}$ & $-0.7(4.9)$ & $-1.6(4.9)$ & $0.1(4.8)$ & .0009 \\
\hline & $\begin{array}{l}\text { Diff base- } \\
\text { line vs V3 }\end{array}$ & $-1.3(5.8)$ & $-2(5.5)$ & $-0.6(6)$ & .0824 \\
\hline \multirow[t]{2}{*}{ PACES omitted } & $\begin{array}{l}\text { Diff base- } \\
\text { line vs V2 }\end{array}$ & $-0.5(5.3)$ & $-1.4(5.2)$ & $0.4(5.2)$ & .0026 \\
\hline & $\begin{array}{l}\text { Diff base- } \\
\text { line vs V3 }\end{array}$ & $-1.2(6.2)$ & $-2.4(5.7)$ & $0.2(6.5)$ & .0042 \\
\hline \multirow[t]{2}{*}{ SMART omitted } & $\begin{array}{l}\text { Diff base- } \\
\text { line vs V2 }\end{array}$ & $-0.4(4.7)$ & $-1.1(4.8)$ & $0.3(4.6)$ & .0157 \\
\hline & $\begin{array}{l}\text { Diff base- } \\
\text { line vs V3 }\end{array}$ & $-0.8(5.2)$ & $-1.7(5.2)$ & $0(5.1)$ & .0476 \\
\hline
\end{tabular}

Abbreviations: Diff, difference; PHQ-9, nine-item Patient Health Questionnaire; V, visit. ${ }^{\mathrm{a}} t$ test.
TA B LE 4 Validation exercise to examine change over time difference in PHQ-9 total scores after sequential omission of each individual randomized controlled trial

\begin{tabular}{|c|c|c|c|c|}
\hline \multirow[b]{3}{*}{ Visit } & \multirow[b]{3}{*}{ Mean (SD) } & \multicolumn{2}{|c|}{ Treatment assignment } & \multirow[b]{3}{*}{$P$} \\
\hline & & Intervention & Control & \\
\hline & & Mean (SD) & Mean (SD) & \\
\hline Baseline, $\mathrm{N}=453$ & $2.9(0.9)$ & $2.9(0.9)$ & $2.8(0.8)$ & 6255 \\
\hline Visit $2, \mathrm{n}=398$ & $2.7(0.8)$ & $2.6(0.9)$ & $2.9(0.8)$ & .0017 \\
\hline Visit $3, \mathrm{n}=255$ & $2.6(0.9)$ & $2.5(0.9)$ & $2.8(0.8)$ & .0045 \\
\hline
\end{tabular}

TA B L E 5 QOLIE-10 mean scores over time in people with epilepsy randomized to self-management versus controls

Abbreviation: QOLIE-10, 10-item Quality of Life in Epilepsy.

people with epilepsy. A recent literature review ${ }^{16}$ found that psychological treatments that encompass a broad range of nonpharmacological interventions for individuals, families, or groups have strong evidence for improving depressive symptoms in epilepsy. However, this review only identified one study that specifically investigated depression outcomes for a self-management intervention. ${ }^{6}$ Our analysis provides additional evidence that self-management support can consistently improve depressive symptom severity in epilepsy. In addition to improving depressive outcomes, self-management support in this pooled analysis was associated with improved quality of life for people with epilepsy.

A recent literature review by Luedke and colleagues ${ }^{17}$ that specifically evaluated epilepsy self-management interventions suggested that changes in knowledge about epilepsy as well as improvement in self-efficacy, self-management skills, and lifestyle modification might explain, at least in part, the reduction in depressive symptoms that may be observed in people with epilepsy who participate in self-management 
TA B L E 6 Mixed-model analysis of the association of baseline demographic and clinical variables with PHQ-9 total score over time in the combined control + intervention group

\begin{tabular}{|lllrr|}
\hline Variable effect & Num DF & Den DF & \multicolumn{1}{l}{$\boldsymbol{F}$} & Pr $>\boldsymbol{F}$ \\
\hline Treatment & 1 & 247 & 20.39 & $<.0001$ \\
\hline Visit & 2 & 247 & 3.83 & .0231 \\
\hline Treatment $\times$ visit & 2 & 247 & 3.05 & .0490 \\
\hline Age & 1 & 247 & 0.00 & .9784 \\
\hline Gender & 2 & 247 & 0.16 & .8536 \\
\hline Race & 2 & 247 & 2.82 & .0613 \\
\hline Ethnicity & 1 & 247 & 0.07 & .7928 \\
\hline Education & 1 & 247 & 7.23 & .0076 \\
\hline Income & 2 & 247 & 0.53 & .5874 \\
\hline Marital status & 1 & 247 & 0.18 & .6711 \\
\hline Seizure frequency & 1 & 247 & 1.26 & .2631 \\
\hline QOLIE-10 & 1 & 247 & 157.10 & $<.0001$ \\
\hline
\end{tabular}

Abbreviations: Den DF, denominator of degrees of freedom; $F, F$ statistic associated with the given source; Num DF, number of degrees of freedom; PHQ-9, nine-item Patient Health Questionnaire; $\operatorname{Pr}>F, P$ value associated with the $F$ statistic of a given source; QOLIE-10, Quality of Life in Epilepsy.

programs. It is possible that the holistic/whole-person focus, which is an intrinsic element of this approach, can help individuals regulate their emotions and make healthy lifestyle changes that enhance mood and well-being. Supportive interactions with self-management interventionists and the group format in some of the programs might also help reduce social isolation and loneliness experienced by some people with epilepsy.

An important feature of the MEW Network applied research agenda is collaboration with community stakeholders, including people with epilepsy, their families, public service agencies, clinicians, and healthcare entities, to scale up and disseminate epilepsy self-management approaches. ${ }^{7,8}$ Consistent with this science-to-service mission, there are a variety of opportunities for clinicians, patients, and families to access MEW Network programs and tools (https://manag ingepilepsywell.org/). Another key element of initiating care and support for people with epilepsy who have depression is early screening and identification. The American Academy of Neurology includes screening for psychiatric and behavioral disorders at each epilepsy care encounter as a quality measure for the delivery of optimal care and better outcomes for individuals with epilepsy. ${ }^{25}$ Effective screening can identify individuals who might benefit from self-management support or other treatments to manage their depression.

An additional clinical implication of our findings relates to the observed trajectory of depression severity in patients with epilepsy who did not receive self-management intervention. In the aggregate data, depression generally did not improve over time. It is possible that the lack of change could have been related to none of the studies being conducted under blinded conditions, and this may have negatively biased outcomes, as people who do not expect to get treatment may not get better. However, the extant literature in chronic diseases generally suggests that depression also tends to be chronic for many individuals, especially if untreated. ${ }^{26,27}$ Our analysis found that individuals with epilepsy who have less education may be particularly likely to have poor outcomes in the usual treatment/wait-list trajectory; this subgroup might benefit from a more intensive and perhaps less complex form of self-management intervention. We did not see that baseline level of education was associated with a difference in depression outcomes among individuals randomized to selfmanagement intervention. This might suggest that self-management intervention could, at least in part, level the playing field or minimize health disparities that might otherwise occur among less educated people with epilepsy.

Our results also highlight the close relationship between depressive symptom severity and quality of life (higher/ worse depression severity $=$ lower quality of life).$^{28}$ The relevance of depressive symptoms to quality of life and other outcomes in people with epilepsy is substantial, and the MEW Network has prioritized a focus on mental health comorbidity with a particular emphasis on depression. ${ }^{9,29}$ A non-MEW Network study that analyzed individuals with poorly controlled epilepsy found that, in order of large to small magnitude, depression, low self-mastery, anxiety, stigma, medical and psychiatric comorbidity, poor medication adherence, and more frequent seizures were associated with worse quality of life. ${ }^{30}$

This analysis has a number of limitations, including the inherent difficulty in interpreting aggregate outcomes from studies that were conducted in different settings with different eligibility criteria. None of the studies required a depression diagnosis or a specific threshold of depressive symptom severity as part of inclusion criteria, and the PHQ-9 is a depression severity instrument that is not specific to epilepsy, in contrast to the epilepsy-specific Neurological Disorders Depression Inventory for Epilepsy. ${ }^{31}$ Calibration needed to harmonize QOLIE data may not have the same validity as the original standardized scale. Studies had follow-up time frequency and durations that were not identical, and the process of harmonizing data from visits for the second and third timepoint follow-up may have minimized important elements of when depression may or may not change over time in people with epilepsy. The control group in our pooled dataset analysis included individuals with epilepsy who were randomized to treatment as usual and those who were randomized to wait-list control, which may have introduced variability to the control group. However, strengths of the data include the relatively large sample, representation of minorities and people with frequent seizures, similarity of 
the demographic and clinical variables among individuals randomized to intervention versus control, and a validation exercise conducted as part of the analysis. Additionally, the PHQ-9 has been selected as an indicator of depression quality of care by the National Quality Forum ${ }^{32}$ and has been widely used in studies of patients with epilepsy. ${ }^{33}$

In conclusion, epilepsy self-management interventions generally address a variety of aspects of helping people with epilepsy learn to manage and cope with this common neurological condition. Aggregate randomized controlled trial findings from a national US epilepsy self-management research collaborative suggest that both depressive symptom severity and quality of life improve with epilepsy self-management approaches. Making self-management interventions more broadly available to people with epilepsy who have depression could potentially improve health generally and minimize the likelihood of complications related to epilepsy.

\section{CONFLICT OF INTEREST}

M.S. has research grants (within the past 3 years) from Otsuka, Alkermes, Janssen, Reuter Foundation, Woodruff Foundation, Reinberger Foundation, National Institutes of Health (NIH), $\mathrm{CDC}$, and International Society of Bipolar Disorders. She has been a consultant to Bracket, Otsuka, Sunovion, Neurocrine, Supernus, and Health Analytics. She has received royalties from Springer Press, Johns Hopkins University Press, Oxford Press, and UpToDate. She has received compensation for continuing medical education activities with American Physician's Institute, MCM Education, CMEology, Potomac Center for Medical Education, Global Medical Education, and Creative Educational Concepts. R.T.F. is a consultant to UCB. B.C.J. has received research grant funding from the Diamond Foundation, Eisai, Medtronic, Neuropace, Sunovion, National Science Foundation, CDC, and Defense Advanced Research Projects Agency. T.M.S. has research grants (within the past 3 years) from UCB, Pfizer, NIH, CDC, and American Heart Association. C.T. has received research grants from the National Science Foundation and Biogen. None of the other authors has any conflict of interest to disclose. We confirm that we have read the Journal's position on issues involved in ethical publication and affirm that this report is consistent with those guidelines.

\section{REFERENCES}

1. Aliasgharpour M, Dehgahn Nayeri N, Yadegary MA, Haghani H. Effects of an educational program on self-management in patients with epilepsy. Seizure. 2013;22:48-52.

2. Ciechanowski P, Chaytor N, Miller J, et al. PEARLS depression treatment for individuals with epilepsy: a randomized controlled trial. Epilepsy Behav. 2010;19:225-31.
3. Dilorio CK, Bamps YA, Edwards AL, et al. The prevention research centers' managing epilepsy well network. Epilepsy Behav. 2010;19:218-24.

4. Thompson NJ, Walker ER, Obolensky N, et al. Distance delivery of mindfulness-based cognitive therapy for depression: project UPLIFT. Epilepsy Behav. 2010;19:247-54.

5. Caller TA, Ferguson RJ, Roth RM, et al. A cognitive behavioral intervention (HOBSCOTCH) improves quality of life and attention in epilepsy. Epilepsy Behav. 2016;57:111-7.

6. Fraser RT, Johnson EK, Lashley S, et al. PACES in epilepsy: results of a self-management randomized controlled trial. Epilepsia. 2015;56:1264-74.

7. Sajatovic M, Tatsuoka C, Welter E, et al. Targeted self-management of epilepsy and mental illness for individuals with epilepsy and psychiatric comorbidity. Epilepsy Behav. 2016;64:152-9.

8. Managing Epilepsy Well Network. Available at: https://manag ingepilepsywell.org/self-management. Accessed May 6, 2019.

9. Sajatovic M, Jobst BC, Shegog R, et al. The Managing Epilepsy Well Network: advancing epilepsy self-management. Am J Prev Med. 2017;52:S241-5.

10. Sahoo SS, Zhang GQ, Bamps Y, et al. Managing information well: toward an ontology-driven informatics platform for data sharing and secondary use in epilepsy self-management research centers. Health Informatics J. 2016;22:548-61.

11. Ramesh P, Wei A, Welter E, et al. Insight: semantic provenance and analysis platform for multi-center neurology healthcare research. Proceedings (IEEE Int Conf Bioinformatics Biomed). 2015;2015:731-6.

12. Friedman D, Spruill TM, Liu H, et al. Depressive symptoms and suicidality among individuals with epilepsy enrolled in self-management studies: results from the US Centers for Disease Control and Prevention Managing Epilepsy Well (MEW) Network. Epilepsy Behav. 2018;87:235-40.

13. Boylan LS, Flint LA, Labovitz DL, Jackson SC, Starner K, Devinsky O. Depression but not seizure frequency predicts quality of life in treatment-resistant epilepsy. Neurology. 2004;62:258-61.

14. Ettinger AB, Good MB, Manjunath R, Faught RE, Bancroft T. The relationship of depression to antiepileptic drug adherence and quality of life in epilepsy. Epilepsy Behav. 2014;36:138-43.

15. Dilorio C, Shafer PO, Letz R, et al. Behavioral, social, and affective factors associated with self-efficacy for self-management among people with epilepsy. Epilepsy Behav. 2006;9:158-63.

16. Michaelis R, Tang V, Goldstein LH, et al. Psychological treatments for adults and children with epilepsy: evidence-based recommendations by the International League Against Epilepsy Psychology Task Force. Epilepsia. 2018;59:1282-302.

17. Luedke MW, Blalock DV, Lewinski AA, et al. Self-management of epilepsy: a systematic review. VA ESP Project \#09-009; 2019. Available at: https://www.hsrd.research.va.gov/publications/esp/ epilepsy.cfm. Accessed August 15, 2019.

18. Noble AJ, Reilly J, Temple J, Fisher PL. Cognitive-behavioural therapy does not meaningfully reduce depression in most people with epilepsy: a systematic review of clinically reliable improvement. J Neurol Neurosurg Psychiatry. 2018;89:1129-37.

19. Caller TA, Secore KL, Ferguson RJ, et al. Design and feasibility of a memory intervention with focus on self-management for cognitive impairment in epilepsy. Epilepsy Behav. 2015;44:192-4.

20. Sajatovic M, Colon-Zimmermann K, Kahriman M, et al. A 6month prospective randomized controlled trial of remotely 
delivered group format epilepsy self-management versus waitlist control for high-risk people with epilepsy. Epilepsia. 2018;59:1684-95.

21. Montgomery SA, Asberg M. A new depression scale designed to be sensitive to change. Br J Psychiatry. 1979;134:382-9.

22. Kroenke K, Spitzer RL, Williams JB. The PHQ-9: validity of a brief depression severity measure. J Gen Intern Med. 2001;16:606-13.

23. Cramer JA, Perrine K, Devinsky O, Meador K. A brief questionnaire to screen for quality of life in epilepsy: the QOLIE-10. Epilepsia. 1996;37:577-82.

24. Cramer JA, Arrigo C, Van Hammee G, Bromfield EB. Comparison between the QOLIE-31 and derived QOLIE-10 in a clinical trial of levetiracetam. Epilepsy Res. 2000;41:29-38.

25. Fountain NB, Van Ness PC, Bennett A, et al. Quality improvement in neurology: Epilepsy Update Quality Measurement Set. Neurology. 2015;84:1483-7.

26. Patten SB. Long-term medical conditions and major depression in a Canadian population study at waves 1 and 2. J Affect Disord. 2001;63:35-41.

27. Gadalla T. Association of comorbid mood disorders and chronic illness with disability and quality of life in Ontario, Canada. Chronic Dis Can. 2008;28:148-54.

28. Tracy JI, Dechant V, Sperling MR, Cho R, Glosser D. The association of mood with quality of life ratings in epilepsy. Neurology. 2007;68:1101-7.
29. Helmers SL, Kobau R, Sajatovic M, et al. Self-management in epilepsy: why and how you should incorporate self-management in your practice. Epilepsy Behav. 2017;68:220-4.

30. Ridsdale L, Wojewodka G, Robinson E, et al. Characteristics associated with quality of life among people with drug-resistant epilepsy. J Neurol. 2017;264:1174-84.

31. Gilliam FG, Barry JJ, Hermann BP, Meador KJ, Vahle V, Kanner AM. Rapid detection of major depression in epilepsy: a multicentre study. Lancet Neurol. 2006;5:399-405.

32. Basch E, Torda P, Adams K. Standards for patient-reported outcome-based performance measures. JAMA. 2013;310:139-40.

33. Gill SJ, Lukmanji S, Fiest KM, Patten SB, Wiebe S, Jetté N. Depression screening tools in persons with epilepsy: systematic review of validated tools. Epilepsia. 2017;58:695-705.

How to cite this article: Sajatovic M, Johnson EK, Fraser RT, et al. Self-management for adults with epilepsy: Aggregate Managing Epilepsy Well Network findings on depressive symptoms. Epilepsia.

2019;60:1921-1931. https://doi.org/10.1111/epi.16322 